\title{
COVID-19 in children with neuromuscular disorders
}

\author{
Daniel Natera-de Benito ${ }^{1}\left(10\right.$ - Sergio Aguilera-Albesa ${ }^{2} \cdot$ Laura Costa-Comellas $^{3} \cdot$ Mar García-Romero $^{4}$. \\ María Concepción Miranda-Herrero ${ }^{5}$. Júlia Rúbies Olives ${ }^{6}$. Óscar García-Campos ${ }^{7}$. Elena Martínez del Val ${ }^{8}$. \\ Maria Josefa Martinez Garcia ${ }^{9}$. Inmaculada Medina Martínez ${ }^{10}$. Ramón Cancho-Candela ${ }^{11}$. \\ Miguel A. Fernandez-Garcia ${ }^{12}$ - Samuel Ignacio Pascual-Pascual ${ }^{4}$. David Gómez-Andrés ${ }^{3}$. Andres Nascimento ${ }^{1,13}$. \\ Neuromuscular Working Group of Spanish Pediatric Neurology Society
}

Received: 22 November 2020 / Revised: 3 December 2020 / Accepted: 4 December 2020 / Published online: 2 January 2021

(c) Springer-Verlag GmbH Germany, part of Springer Nature 2021

\begin{abstract}
Objective Children with neuromuscular disorders have been assumed to be a particularly vulnerable population since the beginning of COVID-19. Although this is a plausible hypothesis, there is no evidence that complications or mortality rates in neuromuscular patients are higher than in the general population. The aim of this study is to describe the clinical characteristics and outcome of COVID-19 in children with neuromuscular disorders.

Methods A registry of children with neuromuscular conditions and laboratory-confirmed-SARS-CoV-2 infection was set up by the Neuromuscular Working Group of the Spanish Pediatric Neurology Society (SENEP). Data to be collected were focused on the characteristics and baseline status of the neuromuscular condition and the course of COVID-19.

Results Severe complications were not observed in our series of 29 children with neuromuscular disorders infected by SARS-CoV-2. Eighty-nine percent of patients were clinically categorized as asymptomatic or mild cases and $10 \%$ as moderate cases. Patients with a relatively more severe course of COVID-19 had SMA type 1 and were between 1 and 3 years.

Conclusions The course of COVID-19 in children with neuromuscular disorders may not be as severe as expected. The protective role of young age seems to outweigh the risk factors that are common in neuromuscular patients, such as a decreased respiratory capacity or a weak cough. Further studies are needed to know if this finding can be generalized to children with other chronic diseases.
\end{abstract}

Keyword Duchenne muscular dystrophy $\cdot$ Myasthenia $\cdot$ Neuropathy $\cdot$ Myopathy $\cdot$ SARS-CoV-2 $\cdot$ Spinal muscular atrophy

\section{Introduction}

Since the end of December 2019, the coronavirus disease 2019 (COVID-19) caused by the severe acute respiratory syndrome virus (SARS-CoV-2) has rapidly spread all over the world, emerging as a global pandemic. Spain, which like many other countries is currently facing the second wave of

The member of Neuromuscular Working Group of Spanish Pediatric Neurology Society are listed in Acknowlegements section.

Supplementary Information The online version contains supplementary material available at https://doi.org/10.1007/s0041 5-020-10339-y.

Daniel Natera-de Benito

dnatera@sjdhospitalbarcelona.org

Extended author information available on the last page of the article the pandemic, has been one of the hardest hit countries so far, recording more than 1,500,000 cases and 41,688 deaths at the time of writing (November 17, 2020) [1]. All the existing studies coincide in pointing out that older people and also individuals with comorbidities are more likely to experience serious and life-threatening complications from COVID-19 [2, 3].

Patients with neuromuscular disorders have been assumed to be a particularly vulnerable population since the beginning of the pandemic [4-6], especially those with low respiratory capacity [7]. Although this is a more than plausible hypothesis, there is no evidence that complications or mortality rates in neuromuscular patients are higher than in the general population. If we specifically focus on the pediatric population with neuromuscular disorders, the available data are even more scarce. Age would be expected to act as a protective factor for this population, since children with 
COVID-19 infrequently have notable disease symptoms [8, 9] but, on the other hand, children with underlying medical conditions seem more susceptible to COVID-19 complications [10-13].

Risk assessment is crucial for identifying patients who are at increased risk of suffering from severe COVID-19 complications. The aim of our retrospective, multicenter study is to describe the clinical characteristics and outcome of COVID19 in children with neuromuscular disorders.

\section{Methods}

A registry of children with neuromuscular conditions and laboratory-confirmed-SARS-CoV-2 infection was set up by the Neuromuscular Working Group of the Spanish Pediatric Neurology Society (SENEP). Irrespectively of clinical signs and symptoms, only patients with a laboratory confirmation of SARS-CoV-2 infection, via real-time reverse-transcription polymerase chain reaction (rRT-PCR) test, serological test and/or antigen test, were included. Data to be collected were defined by the Neuromuscular Working Group of SENEP and were focused on (i) the characteristics and baseline status of the neuromuscular condition and (ii) the course of COVID-19. A request for collaboration was sent to the 486 active members of SENEP (virtually all Spanish pediatric neurologists are members of SENEP). The initial email announcing data collection was sent on October 2, 2020, with a reminder email sent 3 weeks later. Moreover, the records of children hospitalized for COVID-19 in the main Spanish hospitals between March 1, 2020 and November 15, 2020 were reviewed, looking for those who had previously suffered from neuromuscular diseases. Finally, the main Spanish neuromuscular disorders associations (ASEM, Duchenne Parent Project España, FundAME) were informed about this study, and patients with a laboratory-confirmedSARS-CoV-2 infection were invited to contact us to be included in the registry.

Clinical severity of COVID-19 was categorized in five groups: asymptomatic, mild, moderate, severe, and critical, based on previous classifications (Table 1) [14, 15].

\section{Results}

Responses to the call were provided by a large number of hospitals spread throughout Spain, including the 21 centers that have pediatric neurology units accredited by SENEP. A total of 29 individuals under 18 years with a laboratoryconfirmed-SARS-CoV-2 infection were included, of which 20 were males and 9 females. The mean age was 8.4 years, ranging from 4 months to 17 years. The most prevalent neuromuscular condition was spinal muscular atrophy $(11 / 29$, $38 \%$ ), including 6 patients with SMA type 1 and 5 patients with SMA type 2. It was followed by Duchenne muscular dystrophy $(4 / 29,14 \%)$, congenital myopathy $(2 / 29,7 \%)$, congenital muscular dystrophy $(2 / 29,7 \%)$, myasthenia gravis $(2 / 29,7 \%)$, neurogenic arthrogryposis $(2 / 29,7 \%)$, hereditary polyneuropathy $(1 / 29,3 \%)$, PIEZO2-related distal arthrogryposis with impaired proprioception (1/29, 3\%), congenital myasthenic syndrome $(1 / 29,3 \%)$, myotonic dystrophy type $1(1 / 29,3 \%)$, Becker muscular dystrophy $(1 / 29$, $3 \%$ ) and limb-girdle muscular dystrophy (1/29, 3\%). Eight of the patients with SMA were being treated with nusinersen, two with risdiplam and one with salbutamol. The four patients with DMD were undergoing treatment with deflazacort at $0.9 \mathrm{mg} / \mathrm{kg} / \mathrm{day}$. One of the two patients with myasthenia gravis was being treated with pyridostigmine, prednisone and azathioprine, and the other one with pyridostigmine and tacrolimus. The patient with neurogenic arthrogryposis was taking aripiprazole for behavioral difficulties. The clinical characteristics of patients before SARSCoV-2 infection are summarized in Table 2 . The proportion of patients included in our series with severe phenotypes was remarkable: $15 / 29$ patients $(52 \%)$ were wheelchair bound, $12 / 29(41 \%)$ required respiratory support, and 5/29 (17\%) had a gastrostomy tube. By disease group, the proportion of SMA patients who required ventilatory support was particularly high $(9 / 11,82 \%)$. It is worth noting that 9 out of 29 patients $(31 \%)$ had been hospitalized due to respiratory decompensation in the last 5 years, with a range between 1 and 10 admissions (mean 1.2, SD 2.5).

Twenty-six patients (90\%) were diagnosed by PCR and 3 patients $(10 \%)$ by antibody serology testing. Fourteen

Table 1 Categorization of clinical severity in COVID-19

\begin{tabular}{ll}
\hline Asymptomatic infection & Patients with positive SARS-CoV-2 test without any clinical sign or symptom \\
\hline Mild & Patients with symptoms of acute upper respiratory tract infection, including fever, fatigue, myalgia, cough, \\
& sore throat, runny nose, and sneezing without pneumonia \\
Moderate & Patients with pneumonia, frequent fever and cough. Patients with mild respiratory distress, but no respira- \\
& tory deterioration, were included within this group \\
Severe & Patients with respiratory deterioration \\
Critical & Patients with acute respiratory distress syndrome or respiratory failure, shock, or multiple organ dysfunction
\end{tabular}


Table 2 Characteristics of the cohort of children with neuromuscular conditions $(n=29)$ and baseline status before COVID-19

\begin{tabular}{lc}
\hline Male, $n(\%)$ & $20(69 \%)$ \\
Age. mean (range) & $8.4 \mathrm{y}(4 \mathrm{~m}-17 \mathrm{y})$ \\
Neuromuscular condition & \\
Spinal muscular atrophy 1 & $6(21 \%)$ \\
Spinal muscular atrophy 2 & $5(17 \%)$ \\
Duchenne muscular dystrophy & $4(14 \%)$ \\
Other & $14(48 \%)$ \\
Non-ambulant, $n(\%)$ & $15(52 \%)$ \\
Ventilatory support required, $n(\%)$ & \\
NIV, $n$ (\%) & $10(34 \%)$ \\
Tracheostomy, $n(\%)$ & $2(7 \%)$ \\
Gastrostomy tube, $n(\%)$ & $5(17 \%)$ \\
Cardiac involvement, $n(\%)$ & $0(0 \%)$ \\
Cognitive involvement, $n(\%)$ & $4(14 \%)$ \\
Hospitalizations due to respiratory decompensation & $1.2(2.5 ; 0-10)$ \\
in the last 5 years, mean (SD, range) & \\
\hline
\end{tabular}

patients (48\%) were tested after being in close contact with a positive case, 8 patients $(28 \%)$ were tested, because they needed a negative SARS-CoV-2 PCR test prior to medical procedures (spirometry, nusinersen administration, scoliosis surgery) and only 7 patients (24\%) were tested, because they had symptoms. Only 11 of the 29 patients (38\%) had attended school in the 7 days prior to the test. Eleven patients (38\%) remained asymptomatic throughout the course of infection, 16 patients (55\%) presented some mild symptoms, and 3 patients (10\%) with SMA 1 were categorized as moderate, one of them being admitted in the pediatric intensive care unit for 3 days. Fever/low-grade fever $(9 / 29,31 \%)$ and nasal congestion/ rhinorrhea $(9 / 29,31 \%)$ were the most common symptoms. Other less commonly observed symptoms are summarized in Table 3. Apart from one asymptomatic SMA 1 patient who was kept under observation for 3 days and did not require inpatient care, 3 of the 29 patients (10\%) were hospitalized for an average of 7 days (range 3-10 days). These three patients had SMA type 1, were between 1 and 3 years and, based on the clinical severity of COVID-19, were categorized as moderate. Two of the three hospitalized patients had mild respiratory distress without a clear respiratory deterioration (daily hours of non-invasive ventilation were increased from being exclusively nocturnal to being continuous for 3 days, but pressure settings were not modified) and the third developed a pneumonia without respiratory distress. The background treatment regimen was not modified in any of the 29 patients during the infection period.
Table 3 COVID-19 characteristics in children with neuromuscular conditions

\begin{tabular}{lc}
\hline Clinical features, $n(\%)$ & \\
Fever/Low-grade fever & $9(31 \%)$ \\
Nasal congestion/rhinorrhea & $9(31 \%)$ \\
Cough & $3(10 \%)$ \\
Headache & $3(10 \%)$ \\
Myalgia & $2(7 \%)$ \\
Sore throat & $2(7 \%)$ \\
Diarrhea & $1(3 \%)$ \\
Vomiting & $1(3 \%)$ \\
Anosmia & $1(3 \%)$ \\
Pneumonia, $n(\%)$ & $2(7 \%)$ \\
COVID treatment, $n(\%)$ & \\
Oxygen/Increased need for respiratory & $2(7 \%)$ \\
support & $2(7 \%)$ \\
Drugs for COVID-19 & Hydroxychloroquine \\
& Corticosteroids \\
Outcomes, $n(\%)$ & \\
No hospitalization & $26(90 \%)$ \\
Hospitalization & $3(10 \%)$ \\
Intensive care unit & $1(3 \%)$ \\
\hline
\end{tabular}

\section{Discussion}

The clinical spectrum of COVID-19 ranges from asymptomatic to severe pneumonia, acute respiratory distress syndrome and even death. Our study is the first to assess the impact of COVID-19 in children with neuromuscular conditions, a group of patients that has understandably been considered to be highly vulnerable to SARS-CoV-2, despite the absence of reliable data [5-7].

Our results suggest that the course of COVID-19 in children with neuromuscular disorders may not be as severe as expected, although children with SMA 1 appear to be a more vulnerable group. Respiratory complications or need of extra ventilatory support were uncommon in our series, despite the fact that more than $40 \%$ of our patients had severe respiratory involvement prior to SARS-CoV-2 infection (defined as use of non-invasive ventilation for at least $8 \mathrm{~h}$ a day). Except for three cases, all patients presented here were categorized as asymptomatic or mild, similar to what has been previously observed in healthy children $[8$, 10,13]. All three patients who were hospitalized and some other patients who successfully overcame the SARS-CoV-2 infection with hardly any symptoms had a previous history of frequent hospital admissions in the past years due to respiratory decompensations triggered by other infectious agents. According to our data, it does not appear that SARSCoV-2 infection is more life threatening for these children than other viruses such as rhinovirus or influenza. 
Our study has several limitations worth noting. Our method of case capture has proven to be successful, but it is not exhaustive and may not identify all cases. Spanish reports provide a description of COVID-19 cases by age but do not include information about whether an individual who has been infected by SARS-CoV-2 had a neuromuscular condition. In addition, we cannot accurately calculate the incidence of SARS-CoV-2 among Spanish children with neuromuscular disorders, since mild COVID-19 cases have not been systematically tested throughout the pandemic in our country and asymptomatic cases remain unrecognized. Therefore, due to the Spanish testing policy, we assume that many mild cases may have gone unnoticed, unlike moderate, severe or critical cases. This reinforces our idea that COVID-19 in children with neuromuscular conditions may be not as severe as expected.

According to our early results, the protective role of young age seems to outweigh the risk factors that are common in neuromuscular patients, such as a decreased respiratory capacity or a weak cough. We wonder if this finding can be generalized to children with other chronic diseases. The fact that the vast majority of children with neuromuscular diseases overcome SARS-CoV-2 infection without shortterm complications is reassuring news for families and professionals in these times of uncertainty. Despite this, caution must be exercised until our suggestive data can be confirmed and extended in larger cohorts of patients. The results of this study cannot be extrapolated to the adult population. Social distancing should still be the primary advice for all children with neuromuscular disorders and their caregivers to reduce the risk of contracting the virus.

Acknowledgement Ana Camacho:Department of Pediatric Neurology, Hospital Universitario 12 de Octubre, Madrid, Spain. Carlos Ortez: Neuromuscular Unit, Department of Pediatric Neurology, Hospital Sant Joan de Déu and Institut de Recerca Sant Joan de Déu, Barcelona, Spain. Marcos Madruga: Department of Pediatric Neurology, Hospital Virgen del Rocio, Sevilla, Spain. Francina Munell, Mireia Alvarez Molinero: Pediatric Neuromuscular disorders Unit, Pediatric Neurology, Vall d'Hebron University Hospital and Vall d'Hebron Institute of Research (VHIR), Barcelona, Spain. Itxaso Martí: Department of Pediatric Neurology, Hospital Universitario Donostia, San Sebastian, Spain. Inmaculada Pitarch: Department of Pediatrics, Hospital Universitari La Fe, Valencia, Spain. Maria Rosario Domingo Jiménez: Pediatric Neurology Unit, Department of Pediatrics, Hospital Virgen de la Arrixaca, Murcia, Spain. Gema Iglesias Escalera: Department of Pediatrics, Hospital Universitario Puerta de Hierro, Madrid, Spain. Joaquin Fernández Ramos: Department of Pediatrics, Hospital Reina Sofia, Córdoba, Spain. Marta Gómez Garcia-de la Banda: Pediatric Neurology and ICU Department, AP-HP Université Paris Saclay, DMU Santé de l'Enfant et de l'Adolescent, Hôpital Raymond Poincaré, Garches, France. Diana Ghandour: Department of Pediatrics, Hospital Universitario Infanta Elena, Madrid, Spain. María Antonia Grimalt: Department of Pediatrics, Hospital Son Espases, Palma de Mallorca, Spain. Gisela Nogales: Neuromuscular and Neuropediatric Research Group, Germans Trias i Pujol Research Institute, Campus Can Ruti, Universitat Autònoma de Barcelona, 08916 Badalona, Spain; Neuromuscular Working Group of Spanish Pediatric Neurology Society
Data availability Any data not published within the article will be shared, upon reasonable request.

\section{Compliance with ethical standards}

Conflicts of interest None of the authors has any conflict of interest to disclose.

Ethical approval This study was carried out following the ethical guidelines of each of the institutions involved. Written informed consent and age-appropriate assent for study participation were obtained by a qualified investigator [Protocol approved by the Clinical Ethics Committee of Hospital Sant Joan de Déu, Barcelona (PIC 258-20)].

\section{References}

1. Centro de Coordinación de Alertas y Emergencias Sanitarias. Actualización no 252. Enfermedad por el coronavirus (COVID19). Nov 17, 2020. https://www.mscbs.gob.es/profesionales/ saludPublica/ccayes/alertasActual/nCov/documentos/Actualizac ion_252_COVID-19.pdf

2. Zhou F, Yu T, Du R et al (2020) Clinical course and risk factors for mortality of adult inpatients with COVID-19 in Wuhan, China: a retrospective cohort study. Lancet 395:1054-1062. https://doi. org/10.1016/S0140-6736(20)30566-3

3. Luo L, Fu M, Li Y et al (2020) The potential association between common comorbidities and severity and mortality of coronavirus disease 2019: A pooled analysis. Clin Cardiol 43(12):1478-1493. https://doi.org/10.1002/clc.23465

4. Veerapandiyan A, Connolly AM, Finkel RS et al (2020) Spinal muscular atrophy care in the COVID-19 pandemic era. Muscle Nerve 62:46-49. https://doi.org/10.1002/mus.26903

5. Guidon AC, Amato AA (2020) COVID-19 and neuromuscular disorders. Neurology 94:959-969. https://doi.org/10.1212/ WNL.0000000000009566

6. Costamagna G, Abati E, Bresolin N et al (2020) Management of patients with neuromuscular disorders at the time of the SARSCoV-2 pandemic. J Neurol. https://doi.org/10.1007/s00415-02010149-2

7. Damian MS COVID-19 and people with neuromuscular disorders: World Muscle Society position and advice. https://www.world musclesociety.org/files/COVID19/2020-05-11/2020-05-11-WMSCovid-19-advice-v2.pdf (accessed Oct 29, 2020).

8. Parri N, Lenge M, Cantoni B et al (2020) COVID-19 in 17 Italian pediatric emergency departments. Pediatrics. https://doi. org/10.1542/peds.2020-1235

9. Eastin C, Eastin T (2020) Epidemiological characteristics of 2143 pediatric patients with 2019 coronavirus disease in China. J Emerg Med. https://doi.org/10.1016/j.jemermed.2020.04.006

10. Parri N, Magistà AM, Marchetti F et al (2020) Characteristic of COVID-19 infection in pediatric patients: early findings from two Italian pediatric research networks. Eur J Pediatr 179:1315-1323. https://doi.org/10.1007/s00431-020-03683-8

11. DeBiasi RL, Song X, Delaney M et al (2020) Severe coronavirus disease-2019 in children and young adults in the Washington, DC, metropolitan region. J Pediatr 223:199-203. https://doi. org/10.1016/j.jpeds.2020.05.007

12. Chao JY, Derespina KR, Herold BC et al (2020) Clinical characteristics and outcomes of hospitalized and critically ill children and adolescents with coronavirus disease 2019 at a tertiary care medical center in New York City. J Pediatr 223:14-19. https://doi. org/10.1016/j.jpeds.2020.05.006 
13. Bellino S, Punzo O, Rota MC et al (2020) COVID-19 disease severity risk factors for pediatric patients in Italy. Pediatrics 146:e2020009399. https://doi.org/10.1542/peds.2020-009399

14. Lu X, Zhang L, Du H et al (2020) SARS-CoV-2 infection in children. N Engl J Med 382:1663-1665. https://doi.org/10.1056/ nejmc2005073
15. Buonsenso D, Parri N, De Rose C, Valentini P (2020) Toward a clinically based classification of disease severity for paediatric COVID-19. Lancet Infect Dis S 1473-3099:30396. https://doi. org/10.1016/S1473-3099(20)30396-0

\section{Affiliations}

Daniel Natera-de Benito ${ }^{1}\left[\right.$ - Sergio Aguilera-Albesa ${ }^{2} \cdot$ Laura Costa-Comellas $^{3} \cdot$ Mar García-Romero $^{4}$. María Concepción Miranda-Herrero ${ }^{5}$. Júlia Rúbies Olives ${ }^{6} \cdot$ Óscar García-Campos $^{7}$. Elena Martínez del Val ${ }^{8}$. Maria Josefa Martinez Garcia ${ }^{9} \cdot$ Inmaculada Medina Martínez ${ }^{10}$. Ramón Cancho-Candela ${ }^{11}$. Miguel A. Fernandez-Garcia ${ }^{12}$ - Samuel Ignacio Pascual-Pascual ${ }^{4}$. David Gómez-Andrés ${ }^{3}$. Andres Nascimento ${ }^{1,13}$. Neuromuscular Working Group of Spanish Pediatric Neurology Society

1 Department of Pediatric Neurology, Neuromuscular UnitHospital Sant Joan de Déu and Institut de Recerca Sant Joan de Déu Passeig Sant Joan de Déu, 2Esplugues de Llobregat, 08950 Barcelona, Spain

2 Pediatric Neurology Unit, Department of Pediatrics, Complejo Hospitalario de Navarra, Navarrabiomed, Pamplona, Spain

3 Pediatric Neuromuscular Disorders Unit, Pediatric Neurology, Vall d'Hebron University Hospital and Vall d'Hebron Institute of Research (VHIR), Barcelona, Spain

4 Department of Pediatric Neurology, Hospital la Paz, Madrid, Spain

5 Department of Pediatric Neurology, Hospital General Universitario Gregorio Marañón, Madrid, Spain

6 Department of Pediatric Neurology, Hospital Universitari Son Espases, Palma de Mallorca, Spain
7 Department of Pediatric Neurology, Hospital General Universitario de Toledo, Toledo, Spain

8 Department of Pediatrics, Hospital Universitario Fundación Alcorcón, Madrid, Spain

9 Pediatric Neurology Unit, Department of Pediatrics, Hospital Virgen de la Arrixaca, Murcia, Spain

10 Department of Pediatrics, Hospital Universitario Virgen de las Nieves, Granada, Spain

11 Department of Pediatrics, Hospital Universitario Río Hortega, Valladolid, Spain

12 Neuromuscular Service, Department of Paediatric Neurology, Evelina Children's Hospital, Guy's and St Thomas`Hospital NHS Foundation Trust, London, UK

13 Center for Biomedical Research Network On Rare Diseases (CIBERER), ISCIII, Madrid, Spain 